\title{
On the Energy Savings Achieved through an Internet of Things enabled Smart City Trial
}

\author{
Luis Sánchez, Ignacio Elicegui, Javier Cuesta, Luis Muñoz
}

\begin{abstract}
Improving efficiency of city services and facilitating a more sustainable development of cities are the main drivers of the smart city concept. This paper describes a field trial that instantiates a novel architecture exploiting major concepts from the Future Internet (FI) paradigm. The trial has been executed in one of the parks of the city of Santander providing an autonomous public street lighting adaptation service. The trial takes advantage of both the critical communications infrastructures already in place and owned by the utility as well as of the Internet of Things infrastructure belonging to the city municipality to accelerate efficient provision of existing and new city services. The main contribution presented in the paper is, indeed, the assessment of the energy savings achieved during the field trials and the study of key performance indicators analyzed during the trial. The paper highlights how FI technologies create the necessary glue and logic that allows the integration of current vertical and isolated city services into a holistic solution., which enables a huge forward leap for the efficiency and sustainability of our cities. Finally, the trial is a showcase on how added-value services can be seamlessly created on top of the proposed architecture.
\end{abstract}

Index Terms - Future Internet, Smart City, Internet of Things, Field trial, Key Performance Indicators.

\section{INTRODUCTION}

$\mathrm{T}$ HE GROWTH and change in cities is accelerating and makes it even harder to provide a sustainable urban living environment [1]. The use of an Information and Communication Technologies (ICT) based infrastructure alongside the traditional utilities and services infrastructures will be the next big step in the development of cities [2][3]. This revolution is still only at the beginning as suitable infrastructures are being deployed and significant investments into the city infrastructures are made.

Therefore, in this paper we are proposing the smart city community to leverage an innovative Internet of Things (IoT) based architecture aiming at making the traditional utilities' services and infrastructure accessible by different stakeholders (e.g. telco provider, enterprise or 3rd party developers). This

September, $30^{\text {th }}$ 2013. The work described in this paper has been carried out under the framework of the OUTSMART project which has been partially funded by the European Commission under the contract number FP7-ICT285038.

Luis Sanchez, Ignacio Elicegui, Javier Cuesta and Luis Muñoz are with the Communications Engineering Department at University of Cantabria, Santander, $39005 \quad$ Spain (e-mail: 1sanchez@tlmat.unican.es, iemaestro@tlmat.unican.es, jcuesta@tlmat.unican.es, luis@tlmat.unican.es). approach will enable the creation of more transversal and smarter services. Such architecture is additionally biased by the following conditions:

1) It relies on the Future Internet (FI) paradigm for providing ubiquitous services to the users in the cities;

2) It exploits the infrastructures belonging to the utility providers for improving the quality of urban services while reducing the cost of deployment of new infrastructure.

In order to demonstrate the feasibility of the solution proposed we have integrated a reference platform leveraging existing FI enablers. On top of this smart city platform we have built an Autonomous Public Street Lighting Adaptability application that has been used on a field trial in one of the parks of the city of Santander. Additionally, results from the assessment of some Key Performance Indicators (KPI) evaluated during the field trial are provided.

This paper is organized as follows. Section 2 describes the smart city platform architecture that we are proposing. In Section 3 we present the scenario and details of the field trial carried out leveraging prototype implementation following the designed framework guidelines. Both the physical deployment carried out as well as the autonomous light intensity adaptation service that has been developed will be described. The assessment of the results obtained during the field trial realization will be presented in Section 4. Finally, Section 5 concludes this paper.

\section{IOT-ENABLED SMART CITY PLATFORM}

Aiming at harmonizing the different requirements, functionalities and constrains so far described it is desirable to identify a reference model which might guide the IT architects in designing and implementing new city paradigms. From an IT perspective we believe that the model has to follow a four tiered approach and consisting of:

- Capillary networks tier, comprising the heterogeneous underlying infrastructure composed of wireless sensors and actuators as well as proprietary networks belonging to utilities and local authorities.

- Network backbone tier, which interconnects every capillary network and provides southbound homogenization.

- Enabling technologies tier, which holds future internet components exporting infrastructure capabilities on a uniform manner.

- Services and applications tier, where platform services are offered to application developers. 
Through this group of layers, one of the main targets of this approach is to provide access to application developers to the set of heterogeneous data supplied by different sensor networks and legacy systems, in an open and standardized way, completely abstracting these two worlds. The different functionalities offered at each of these tiers are presented in the following sub-chapters.

\section{A. Capillary networks and utility legacy systems}

At the bottom of the architecture, heterogeneous infrastructure is supported. It is important to highlight that this layer provides the necessary substrate consisting of IoT devices and the communications networks that allow bidirectional access to the underlying infrastructure.

The key aspect that differentiates this reference model from other existing IoT ones [4] is that it explicitly incorporates the utilities and local authorities' networks as part of it. In this sense, the heterogeneity introduced by these networks poses challenges that must be addressed on the upper layers so that underlying heterogeneity can be managed on a homogeneous way at application level as well as within the platform itself.

\section{B. Network backbone}

Underlying heterogeneity is hidden on the network backbone tier. Capillary network access gateways and protocol adapters are responsible for assuring communication unification and reliability. Additionally, data transformation is realized at this level. Finally, device and resources traceability is initiated within the network backbone access gateways.

In this sense, within the data plane, standardized data model (e.g. based on SensorML [5]) is applied to transform the information coming from the subjacent infrastructure on its proprietary format into a standardized format used within the data processing enabling technologies.

Moreover, within the network backbone tier, control plane functionalities are also addressed. By using a common resource model (e.g. based on eXtensible Markup Language (XML) or Resource Description Framework (RDF)) it is possible to query and retrieve these resources. These network management mechanisms carried out at capillary network access gateways are able to realize the resource discovery process. The selection of suitable resources with particular context attributes such as locality or sampling frequency and that capture certain phenomena occurring in the environment is also supported by using this resource model to uniformly describe their attributes, capabilities and roles.

Finally, the resource monitoring process concerns the dependability of the testbed platform i.e. its robustness with respect to software component or equipment failure.

\section{Enabling technologies}

If the network backbone layer provides the ways to collect data and control the nodes from the capillary networks and legacy systems, the set of enablers on this tier will focus on the data mining and infrastructure management, providing functionalities related with the data and the management plane. It makes up the core of the proposed architecture, providing also the tools for the upper layer to access data and nodes capabilities.

Related to management plane, the main functionalities provided at this tier are: (1) To support registration of IoT devices (sensors/actuators) and IoT gateways; (2) To enable the backend to control and access information from IoT gateways and IoT devices; and (3) To provide access to information of the different devices registered into the platform (status, location, manufacturer, measurements...) upon request from external management applications.

In the data plane, focused on data collection and storing, the basic functionalities provided are: (1) To support measurements collection and storage processes for different phenomena; (2) To provide mechanisms for upper layers applications to access data collected (historic data and last measurements); (3) To enable the submission of commands to devices; and (4) To implement a publish-subscribe-notify model, allowing rules for subscription to specific events, conditions or devices and the definition of alerts.

The definition of this layer and the enablers initially included have been done following the FI-WARE project guidelines [6] which, aside from giving a more standardized and portable vision of the whole architecture for smart cities, allows that new enablers can be easily integrated into a final deployment, adding new functionalities as event processing, big data analysis and so on.

\section{Service and applications layer}

Based on the interfaces given by the enabling technologies, this tier provides with the development tools needed to create new services which exploit the information gathered from the sensor networks and utility systems, as well as allowing interaction with the deployed actuators.

It also includes the runtime environment with the application server responsible for executing these services and the set of plugins and enablers (service repository, operative systems oriented tools, development libraries, etc.) to complete the service infrastructure.

This new set of services is created and supported by the smart city. This interface is completely independent from the protocols on the data gathering layers so allows creating new open and portable final applications to improve citizen's life quality in smart cities.

Finally, it is important to highlight that services provide another abstraction level to application developers which can reuse the functionalities provided by these services in an abstracted manner while generating applications with addedvalue for the end-users.

\section{FIELD TRIAL SCENARIO}

The objective of the field trial carried out is to demonstrate the applicability of the proposed architecture and assess the capacity of IoT technologies to address smart city challenges and provide relevant savings that leverage the smart city rollout through smart city platforms as the one presented in this paper.

The field trial is focused on the public street lighting system. The Santander City Council spends every year around 
$3,5 \mathrm{M} €$ in streetlights (around a $5 \%$ of its whole goods and services budget and similar budget dedicated to the R\&D), which is, together with urban transport, water and gas distribution, waste management, or infrastructure maintenance, one of the main (and basic) public services this Council manages.

Taking this into account an Autonomous Public Street Lighting Adaptability application was implemented on top of a prototype implementation of the smart city platform reference model described in Section 2.

\section{A. Autonomous Public Street Lighting Adaptability}

The Autonomous Public Street Lighting Adaptability use case aims at increasing the public streetlight service efficiency by automatically adapting the power supplied to the lampposts and their corresponding light level to the current needs of the area covered.

Depending on the city area and the main use of the streetlights, the phenomenon (or set of parameters) chosen to increase/decrease the illumination level may vary (e.g. weather conditions, existing illumination levels, vehicular traffic, etc.). The prototype deployed is focused on pedestrian presence; this is, once the streetlight has been turned on (to its normal level), if no pedestrian presence is detected, the power supplied by the controller will be set to "saving mode" and the light level will decrease. When presence is noticed, automatically the command to activate the normal mode again is sent to the controller, till no one is detected in the area. This operating mode requires a sensor network including presence detector nodes to be deployed in the area covered by the prototype. These nodes will also build in a light sensor, intended to measure the ambient light level. The information provided by this sensor will be used in a twofold manner: when ambient light falls below a fixed level (e.g due to

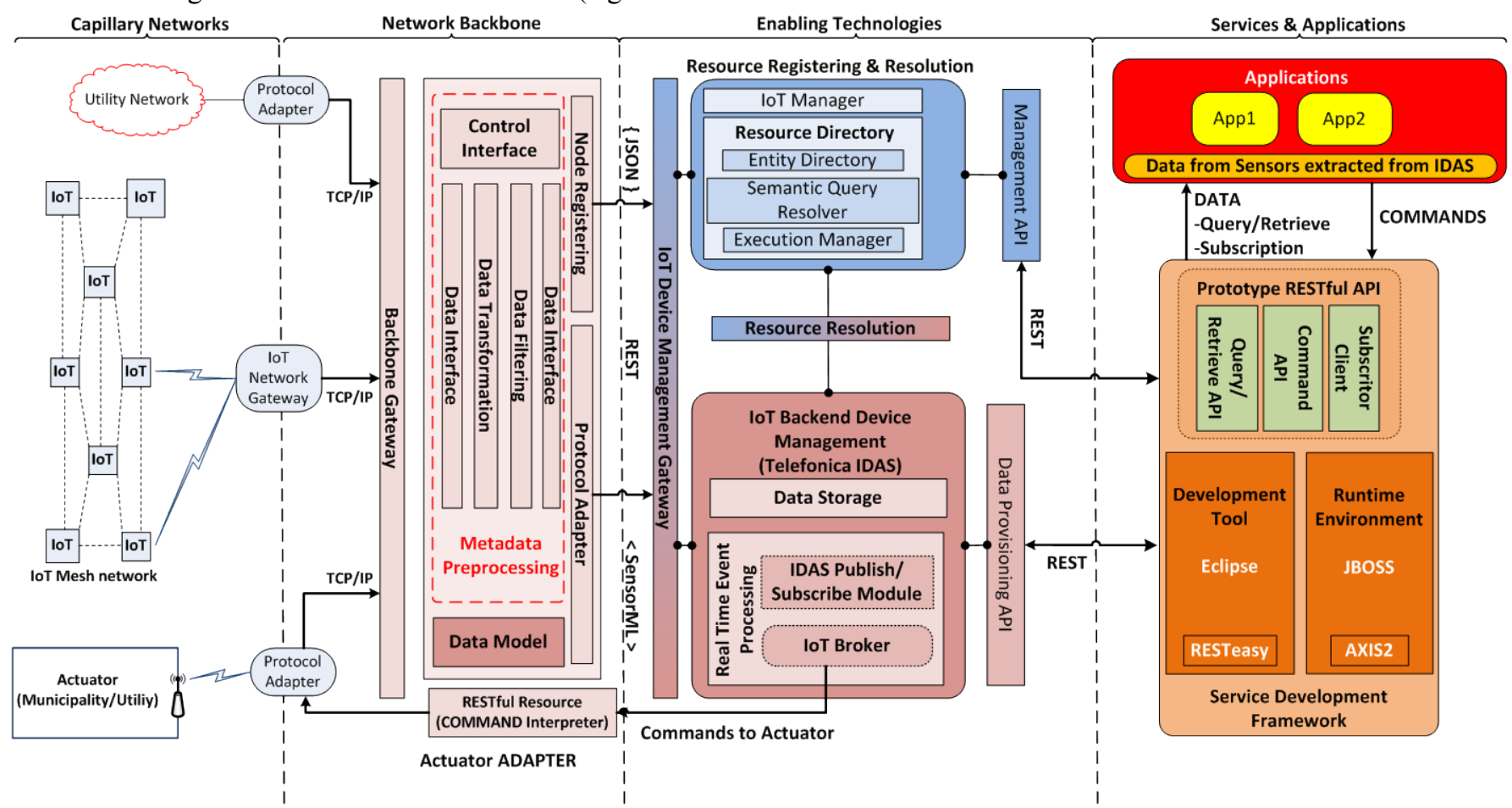

weather conditions), regardless of the astronomical clock of the power controller, the streetlights can be switched on and presence detection mechanism be activated; apart of this, the light sensors installed covers a range from 0 to 100 luxes, which makes them suitable (properly positioned) to evaluate light pollution.

\section{B. Reference model prototype implementation}

The prototype architecture consists on a concrete implementation of the smart city platform proposed in Section 2. Fig. 1 shows the realization of the prototype based on the generic architecture.

Capillary Networks tier includes the Advanced Metering and Management System (AMMS) from the utility infrastructure, a network of wireless sensor nodes, and the power controller as an actuator.

AMMS device, belonging to E-On Spain, is adapted and directed to the Metadata Preprocessing. It uploads daily information about active and reactive power consumption.

IoT Nodes include both one light intensity sensor and one presence detector. The first one provides illumination level in luxes so that pedestrian detection is only enabled during night. The second one triggers an event upon pedestrians are detected. Presence detectors are based on X-Band radar. Twelve of these nodes have been deployed around the pathways in the park. Using a wireless communication module based on IEEE 802.15.4 transceiver, these nodes create a mesh network in which all cooperate for relaying the information in order to reach the IoT Network Gateway. This latter one sends all data collected from nodes to the Metadata Preprocessing component, using a $3 \mathrm{G}$ connection, passing through the Backbone Gateway.

Fig. 1. Deployed prototype architecture 
The Intelligent Control Module of the Power Controller (owned by the local authority) processes and executes all commands received (e.g. commands to switch on/off the streetlights or to change to normal/saving mode). Due to the scale of the field trial, only one power controller is involved. The protocol adapter created to connect with the power controller uses a GSM modem to convert from Modbus ASCII to TCP/IP and reach the Backbone Gateway. At the Network backbone tier three functionalities are realized.

The backbone gateway and Metadata Preprocessing module work as the point where all data sources must send their observations. The backbone gateway receives data from different sources and in different formats: the AMMS works with text comma-separated-value files; IoT mesh network sends proprietary-formatted frames; and the power controller receives, processes and sends through a Modbus ASCII channel. This data is adapted to be correctly accepted by the corresponding enabler on the next layer. The IoT Backend Device Management module process observations in SensorML (XML) while and the Resource Directory uses JSON for nodes' registering and management.

Power Controller Adapter has been implemented as a middleware that abstracts the proprietary protocols and mechanisms of the advanced power controllers used in this deployment. This middleware implements a RESTful API that provides access to controller's information and commands to third parties' software, turning this legacy device into an IoT Node that can be dealt with as a sensor and/or actuator and be easily integrated into FI and smart cities deployments. Upon reception of the commands from the upper tier (as PUT and GET web services), the adapter implements the command conversions to be sent to the power controller in its Modbus protocol. Enabling Technologies has been implemented according to the core platform developed in FI-WARE project. This alignment ensures our prototype to be scalable, portable and compatible with other sensors, services and functionalities. Two enablers, from the FI-WARE catalogue have been used in our prototype:

Resource Directory asset, instantiated on the prototype server, registers all information about nodes' capabilities.

IoT Backend Device Management General Enabler, implemented through an instance of the Telefonica IDAS [7], which collects and stores all observations sent by the capillary networks through the IoT Backend Device Gateway. This element can access to the data stored in the Resource Directory instance and interact with it. The Backend Device Management enabler provides a RESTful API for the service development layer to access the current and historic data supplied by nodes in the capillary network. It also allows registering a service to a node or a set of data (through the publish/subscribe element), so a notification can be sent by the enabler when any change is raised (e.g. when a pedestrian is detected - service registered to presence data). This API also allows sending commands if the final node has been registered as an actuator (e.g. authorized services can operate on IoT Nodes - activate the power controller or change its operating mode).

Services \& Applications tier exploits the functionalities provided by the IoT Backend Device enabler's API to create a set of specific services that conform an easy interface to third parties to create applications based on data and capabilities offered by the deployed capillary networks and legacy systems. In order to support scalability, composition and standardization, this layer has also been developed following the guidelines established in FI-WARE for applications and services delivery framework.

\section{Field trial scenario and contour conditions}

The field trial was run on the Las Llamas Park in Santander. Fig. 2 shows the area where the trial was carried out and some details on the sensor nodes and power controller.

Twelve presence detectors were placed along a 400 meters trail. Each of them was covering around 30 meters of the pathway. Table 1 shows the conditions during the trials.

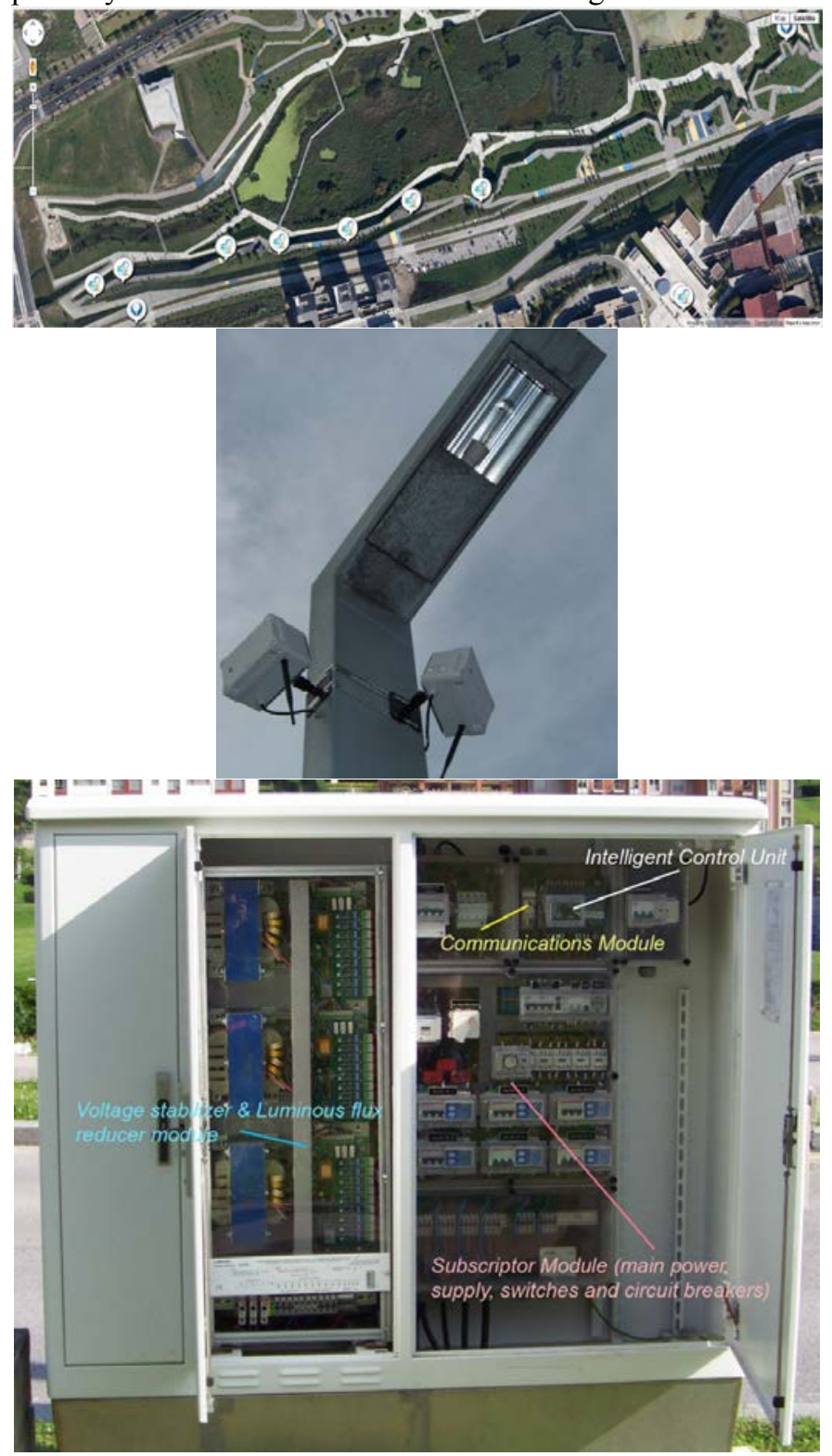

Fig. 2. Details of the field trial deployment 
TABLE I

TRIAL SCENARIO CONDITIONS

\begin{tabular}{|l|l|}
\hline \multicolumn{2}{|c|}{ General Conditions } \\
\hline Test start time & $\begin{array}{l}\text { At dusk according to Observatorio } \\
\text { Astronómico Nacional and Instituto } \\
\text { Geográfico Nacional. }\end{array}$ \\
\hline Test end time & $\begin{array}{l}\text { At Dawn according to Observatorio } \\
\text { Astronómico Nacional and Instituto } \\
\text { Geográfico Nacional. }\end{array}$ \\
\hline Control & $\begin{array}{l}\text { Fully automatic. No manual operation } \\
\text { should take place. }\end{array}$ \\
\hline \multicolumn{2}{|l|}{ Regulator conditions } \\
\hline Restrictions & Utility restrictions to power control apply \\
\hline
\end{tabular}

Santander public streetlights are configured such that electric power controllers, assisted by an astronomical clock, set the power level supplied to a line of streetlights. Thus, the streetlights are turned on at its normal mode (maximum power supplied) at sunset and switched off at sunrise.

The power controller operating mode has been configured to work with the currently installed high pressure sodium lamps. Any change to the voltage supplied to the streetlights (including switching on/off the electrical supply) must be done respecting strict time constraints. In practice, due to sodium lamps thermal stabilization, a 15 minutes guard time slot was established. That means that there should be at least this time between dim up or down commands. Upon sodium lamps replacement by LEDs, these guard time slots could be omitted, and light adaptability would be done almost in real time.

Prototype deployment allowed to test and to evaluate the performance of all components under real conditions. The know-how gained while operating was used for identify the following risks and potential enhancements:

1) Presence detector movement cancellation: wind and people leaning to lampposts can cause the presence detector to tremble. In such cases the presence detector perceives its neighborhood as if it all is shaking, what triggers the presence detection as a false positive. To neutralize this false positives, accelerometer data was included into the detection system, so sensor nodes are able to detect any shake and therefore reject the corresponding detection patterns.

2) Weather conditions: rain affects the behavior of the RADAR presence detection sensors. We identified that it reduces the maximum detection range of the devices and negatively affect the chances of presence detection even when the target is within range. Wet umbrellas and raincoats also reduce the chances of detection. Passive infrared sensors present also troubles when detecting wet targets or working under rainy weather.

3) Maximum network load: network capacity test was performed just after the deployment of the prototype devices; test demonstrated that the network is able to sustain $1 \mathrm{~KB} / \mathrm{s}$ of data traffic with the desired $99.994 \%$ successful data transfer rate. It is possible to achieve higher data transfers rates but collisions turn into some information packets being lost. While this means that it is possible to achieve higher overall data transfers over the network, it also means that some data would be lost in the process. $1 \mathrm{~KB} / \mathrm{s}$ is enough for transferring about 20 data frames per second. Considering that a single network device transmits on average once every 5 minutes, it theoretically means a single gateway should be able to sustain up to 6.000 devices. However, the high correlation between devices sending information poses a problem. As people make use of same public areas at the same time, when some sensor is detecting presence, there is a high chance of other sensors doing so in a small frame of time, generating bursts of data when there is a high usage of the area and large periods of silence when there is no presence. Another limitation on the estimation of the maximum devices is the area usage. If the sensor network is deployed in areas with more pedestrian presence, sensor would send more data, reducing the maximum possible number of devices per gateway.

\section{TRIALS KEY PERFORMANCE INDICATORS ASSESSMENT}

Field trial evaluation took place from April to June 2013. During this period of time the Autonomous Public Street Lighting Adaptability application was running. This transition from early spring time to summer time allowed covering a wide range of different weather conditions.

All data used for compiling these trial results were gathered on the prototype deployment during night conditions and based on the real usage of the area of deployment.

Fig. 3 shows how the system adapts the power served to the lampposts. At dusk, full power is served to the lampposts. Upon detecting the absence or presence of people around the field trial scenario, power is reduced or increased thus dimming down and up the light intensity. Fig. 3a presents a typical summer night with warm weather conditions while Fig. $3 \mathrm{~b}$ shows the power consumption behavior during a rainy night. The application is much more effective on this latter case where, due to the weather conditions, the park was not visited during the whole night.

The following KPIs were considered for evaluating the performance of the prototype deployment:

- Power reduction: measures the enhancement in the city sustainability by measuring the cost reduction achieved due to diminishing lighting levels when there is no people in the trial area.

- Emissions Reduction: measures the enhancement in the city sustainability by measuring the yearly reduction in $\mathrm{CO}_{2}$ emissions by comparing energy consumption before the trial and during it.

- Reactive power: measures technical improvement in efficiency by measuring the variation in reactive versus active power ratio during the trial.

- Service Usefulness: provides the amount of time the system sets the lamppost to saving power level and the amount of times that the service is activated by presence detection.

Table 2 summarizes the mean results obtained during the field trial realization. 
TABLE II

FIELD TRIAL KEY PERFORMANCE INDICATORS EVALUATION SUMMARY

\begin{tabular}{|c|c|c|c|}
\hline KPI & Unit of measurement & Expression & Evaluation \\
\hline $\begin{array}{l}\text { Power reduction } \\
(\mathrm{PR})\end{array}$ & $\begin{array}{l}\text { Normalized power } \\
\text { provided referred to power } \\
\text { provided prior to the trial. }\end{array}$ & $\begin{array}{l}\quad P R=\frac{P 1}{P 0+P 1} * 100 \\
\text { P0: Power used before trial } \\
\text { P1:Power used during trial }\end{array}$ & Normalized power: $27.2 \%$ \\
\hline $\begin{array}{l}\mathrm{CO}_{2} \text { Emissions } \\
\text { Reduction (ER) }\end{array}$ & $\begin{array}{l}\text { Kilograms }(\mathrm{Kg}) \text { of } \mathrm{CO}_{2} \\
\text { emissions }\end{array}$ & $\begin{array}{c}E R=P R * C O 2 \_ \text {Rate } \\
\text { (Considering Spanish generation mix 2011) }\end{array}$ & $\begin{array}{l}\text { Almost } 1 \mathrm{Kg} \text { of } \mathrm{CO}_{2} \text { emissions } \\
\text { savings. }\end{array}$ \\
\hline $\begin{array}{l}\text { Reactive Power } \\
\text { Ratio }\left(\frac{R}{A}\right)\end{array}$ & $\begin{array}{l}\text { Percentage calculated } \\
\text { taking into consideration } \\
\text { the reactive/active power } \\
\text { rate. }\end{array}$ & $\left(\frac{R}{A}\right)=\frac{R P}{A P} * 100$ & $\begin{array}{l}\text { During normal operation: } \\
\qquad\left(\frac{R}{A}\right)=46.89 \% \\
\text { During saving mode: } \\
\qquad\left(\frac{R}{A}\right)=33.10 \%\end{array}$ \\
\hline $\begin{array}{l}\text { Service } \\
\text { Usefulness }\end{array}$ & $\begin{array}{l}\text { Hours and number of times } \\
\text { of presence detection. }\end{array}$ & $\begin{array}{l}\text { Time that service is on saving mode. } \\
\text { Times that the service was demanded. }\end{array}$ & $\begin{array}{l}191 \text { minutes/night } \\
23.5 \text { demands } / \text { night }\end{array}$ \\
\hline
\end{tabular}

Taking into account the results obtained, it is clear that, although the main aim of the smart city prototype implementation was to provide a real-world reference on the applicability of FI technologies in general and IoT in particular to the smart city concept, the field trial has demonstrated that relevant savings have been achieved. As a matter of fact, viability business models estimated a minimum of $10 \%$ savings, in terms of power reduction, for having a viable application. Moreover, Spanish regulation requires a reactive versus active power ratio not higher than $50 \%$.

\section{CONCLUSIONS}

This paper provides insights on the way FI technologies create the necessary glue and logic that allows the integration of current vertical and isolated city services into a holistic solution. It has presented the functional architecture that harmonizes the different requirements imposed by the smart city scenario.

However, the main contribution of the paper is the description of a field trial that has been carried out over a reference prototype implementation. This prototype instantiates the aforementioned architecture. It has been deployed in a real-world environment and targets an application of FI technology with large savings opportunities.

The experiences carried out in this trial have allowed us to

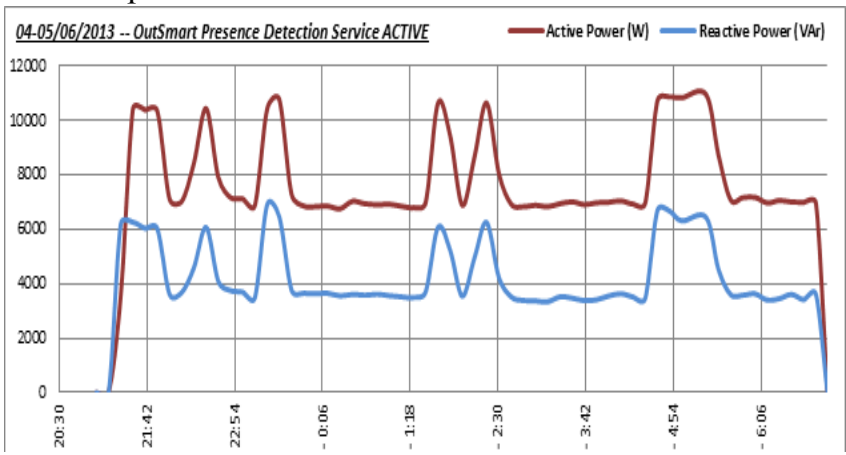

(a) assess several KPI. The summary of this assessment has been presented in the article demonstrating that IoT technologies are able to provide relevant savings on a smart city scenario.

Finally, it is important to highlight that the prototype implementation has targeted the realization of the field trial but it paves the way for the integration of heterogeneous city infrastructure by means of the developed IoT technologies.

\section{REFERENCES}

[1] "Urbanization and health", Bulletin of the World Health Organization, Volume 88, Number 4, April 2010, pp. 245-246

[2] Schaffers, H., Komninos, N., Pallot, M., Trousse, B., Nilsson, M., Oliveira, A. (2011). Smart Cities and the Future Internet: Towards Cooperation Frameworks for Innovation. In: J. Domingue et al. (Eds.), The Future Internet, Springer, pp 431-446.

[3] Hernández-Muñoz, J.M., Vercher, J.B., Muñoz, L., Galache, J.A., Presser, M., Hernández Gómez L. A., and Pettersson, J. (2011). Smart Cities at the Forefront of the Future Internet. In: J. Domingue et al. (Eds.), The Future Internet, Springer, pp 447-462.

[4] Serbanati, A., Medaglia, C. M., \& Ceipidor, U. B. (2011). Building blocks of the internet of things: State of the art and beyond. Deploying RFID-Challenges, Solutions, and Open Issues, C. Turcu, Ed., ed: InTech.

[5] Botts, M. (2004). Sensor Model Language (SensorML) for in-situ and remote sensors. OGC document reference number.

[6] FI-WARE, Future Internet Core Platfom. Available online: http://www.fi-ware.eu.

Telefonica IDAS' FI-WARE wiki. Available online: http://forge.fiware.eu/plugins/mediawiki/wiki/fiware/index.php/IDAS

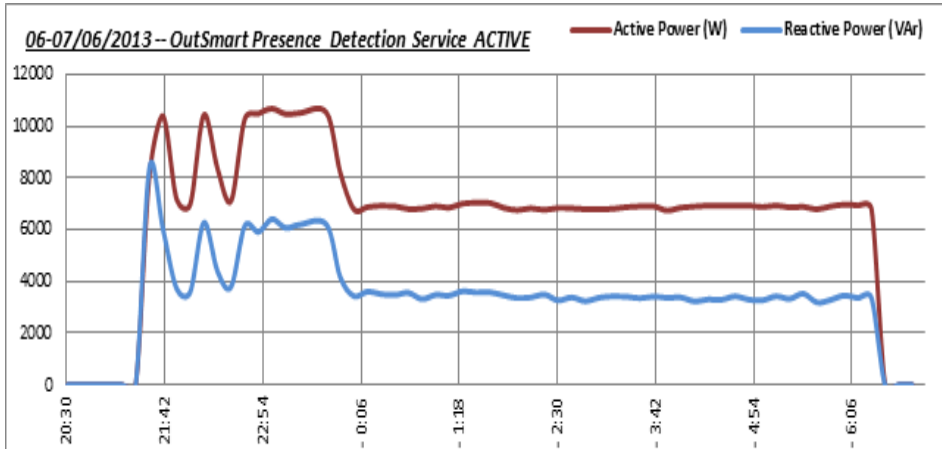

(b)

Fig. 3. Active and reactive power evolution during typical summer night (a) and rainy night (b) 